\title{
Larva de quinto estádio e pupa de Opsiphanes quiteria meridionalis Staudinger (Lepidoptera, Nymphalidae, Brassolinae) ${ }^{1}$
}

\author{
Mirna Martins Casagrande ${ }^{2} \&$ Olaf Hermann Hendrik Mielke 3
}

${ }^{1}$ Contribuição ${ }^{\circ} 1556$ do Departamento de Zoologia, Universidade Federal do Paraná.

${ }^{2,3}$ Departamento de Zoologia, Universidade Federal do Paraná. Caixa Postal 19020, 81531-980 Curitiba-PR, Brasil. Bolsistas CNPq.

\begin{abstract}
Fifth larval instar and pupa of Opsiphanes quiteria meridionalis Staudinger (Lepidoptera, Nymphalidade, Brassolinae). Descriptions of the fifth instar and pupa of Opsiphanes quiteria meridionalis Staudinger, 1877 from material reared in southern Brazil are presented. The immatures, except egg and the adult are illustrated. The larvae feed on leaves of Syagrus romanzoffiana (Cham.) Glassman (Arecaceae).
\end{abstract}

KEYWORDS. Brassolini, hostplant, immature stages.

RESUMO. Larva de quinto estádio e pupa de Opsiphanes quiteria meridionalis Staudinger (Lepidoptera, Nymphalidae, Brassolinae). É apresentada a descrição da larva de quinto estádio e pupa de Opsiphanes quiteria meridionalis Staudinger, 1877 de material proveniente do sul do Brasil. Os imaturos, exceto ovo, e o adulto são ilustrados. Larvas alimentamse de Syagrus romanzoffiana (Cham.) Glassman (Arecaceae).

PALAVRAS-CHAVE. Brassolini, estágios imaturos, planta hospedeira.

Esta contribuição tem como finalidade ampliar o conhecimento de imaturos de lepidópteros neotropicais. O conhecimento desta fase da vida dos insetos tem trazido aos especialistas dedicados ao estudo da classificação de Lepidoptera em seus níveis superiores e suas relações, dados que acrescem a fundamentação de propostas como em Ackery et al. (1999) e Freitas \& Brown Jr. (2004) que não somente fazem uso de carateres de coloração como principalmente àqueles relacionados à morfologia, planta hospedeira $\mathrm{e}$ imaturos.

Casagrande $(1979,2002)$ e Casagrande \& Mielke (2000 a, b; 2003) têm publicado dados sobre imaturos de Brassolinae, porém para Opsiphanes Doubleday, [1849] os registros são escassos e restritos a comparações com outros gêneros (DeVries 1987), estudos filogenéticos (Freitas \& Brown Jr 2004) ou à citação de planta alimentícia (Ackery 1988).

\section{MATERIAL E MÉTODOS}

As larvas, alimentadas com folhas de Syagrus romanzoffiana (Cham.) Glassman (Arecaceae), foram mantidas em casa de vegetação em São Bento do Sul, Santa Catarina, até a emergência dos adultos. Os adultos que emergiram estão depositados na Coleção Entomológica Pe. Jesus Santiago Moure, Departamento de Zoologia, Universidade Federal do Paraná (DZUP). O exemplar fêmea que ilustra este artigo é proveniente desta criação, porém o macho é de Joinville, Santa Catarina. Cápsulas cefálicas, larvas de quinto estádio e pupas estão preservados em álcool 70\%.
Larva de quinto estádio

(Figs. 1-6)

Cabeça de textura irregular, forma quadrangular, comprimida dorso-ventralmente com quatro pares de escolos, formando uma coroa na parte posterior da cabeça. Coloração geral branco leitosa, com dois pares de faixas cinza: uma lateral, desde a base do anel de estemas até o meio do primeiro e segundo par de escolos; outra, anterior, desde o centro da sutura epicranial até a base do par de escolos dorsais. Sutura epicranial com a mesma cor das faixas. Pares de escolos dorsais e dorso - laterais de um laranja intenso com extremidade preta. Base do par dorsal, com uma mancha arredondada preta. Terceiro par, lateral, preto e aproximadamente um terço do tamanho do anterior e o quarto, látero-ventral, como uma pequena rugosidade, também preto. Com quatro estemas láterodorsais e dois ventrais; base do segundo e quarto estemas preto, quinto cinza, e primeiro e sexto com anel branco. Cerdas translúcidas distribuídas por toda cabeça. Face ventral cinza.

Tórax e abdome pubescentes de coloração geral verde intenso. Área dorsal com faixa mediana verde, marginada por verde mais claro. Área subdorsal cinza, quase preto, com cerdas translúcidas sobre calazas branco leitosas. Área supraespiracular de igual aspecto a subdorsal, porém dois terços da largura desta e, área espiracular com a mesma coloração das anteriores, não contínua e metade da largura da supraespiracular. Entre as áreas subdorsal, supra-espiracular e espiracular, áreas de cor verde como a faixa mediana, também com cerdas translúcidas sobre calazas branco leitosas. 

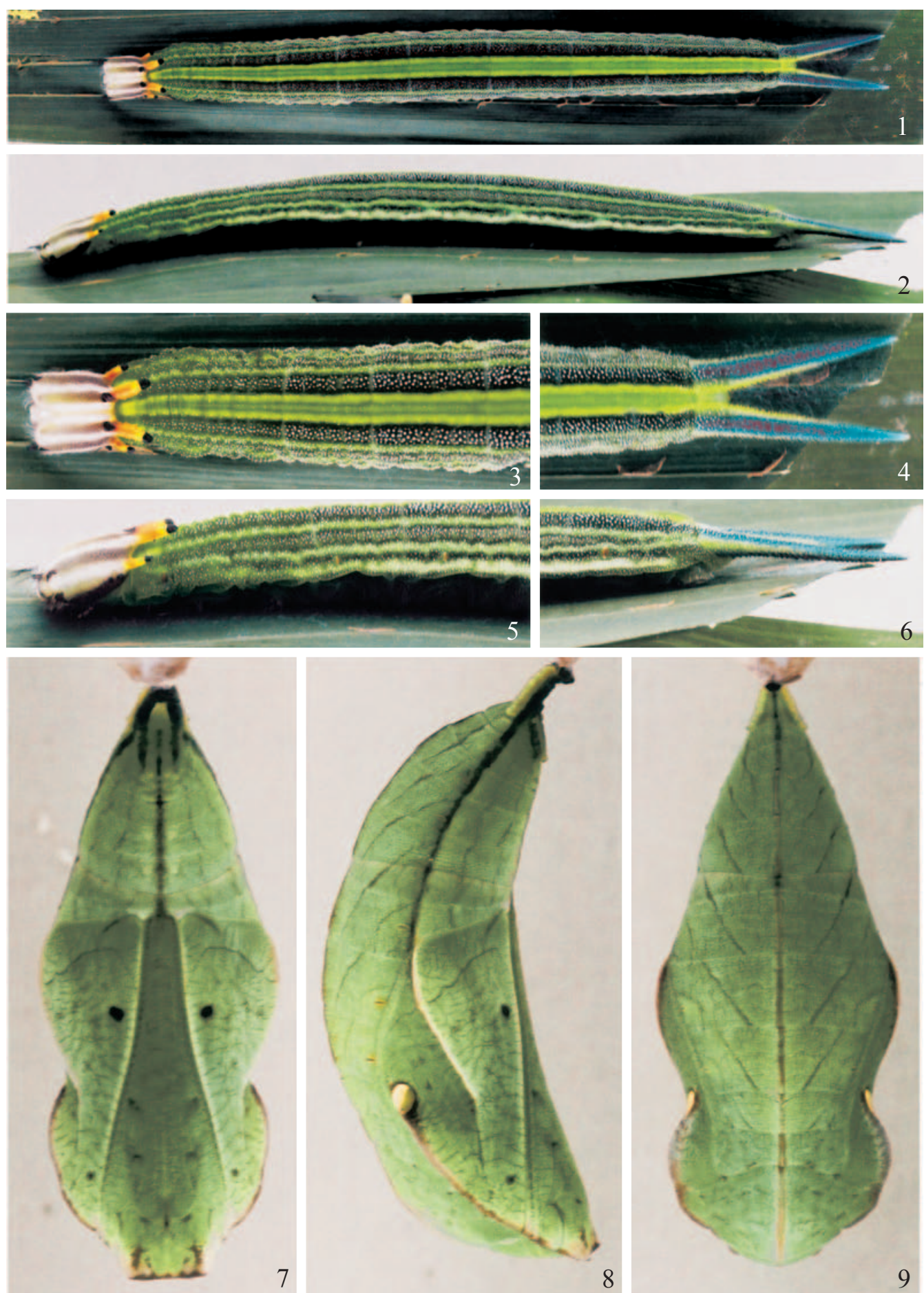

Figs. 1- 9. Opsiphanes quiteria meridionalis Staudinger. Larva de quinto estádio. 1, vistas dorsal; 2, vista lateral; 3, vista dorsal da cabeça, tórax e parte do abdome; 4, vista dorsal da placa suranal; 5, vista lateral da cabeça, tórax e parte do abdome; 6, vista lateral da placa suranal. Pupa. 7 , vista anterior; 8 , vista lateral; 9, vista posterior.

Projeções da placa suranal verdes, como na área dorsal, dorsalmente verde azulado, como uma continuação da mesma cor da área subdorsal. Peritrema dos espiráculos torácico e abdominais com a mesma cor laranja intensa dos escolos dorsais e dorso-laterais. Face ventral do tórax e abdome verde musgo com cerdas translúcidas em calazas branco leitosas. Pernas torácicas pretas e abdominais com a mesma coloração do tegumento.

As larvas atingem um comprimento máximo de $10 \mathrm{~cm}$, incluindo as projeções da placa suranal. 

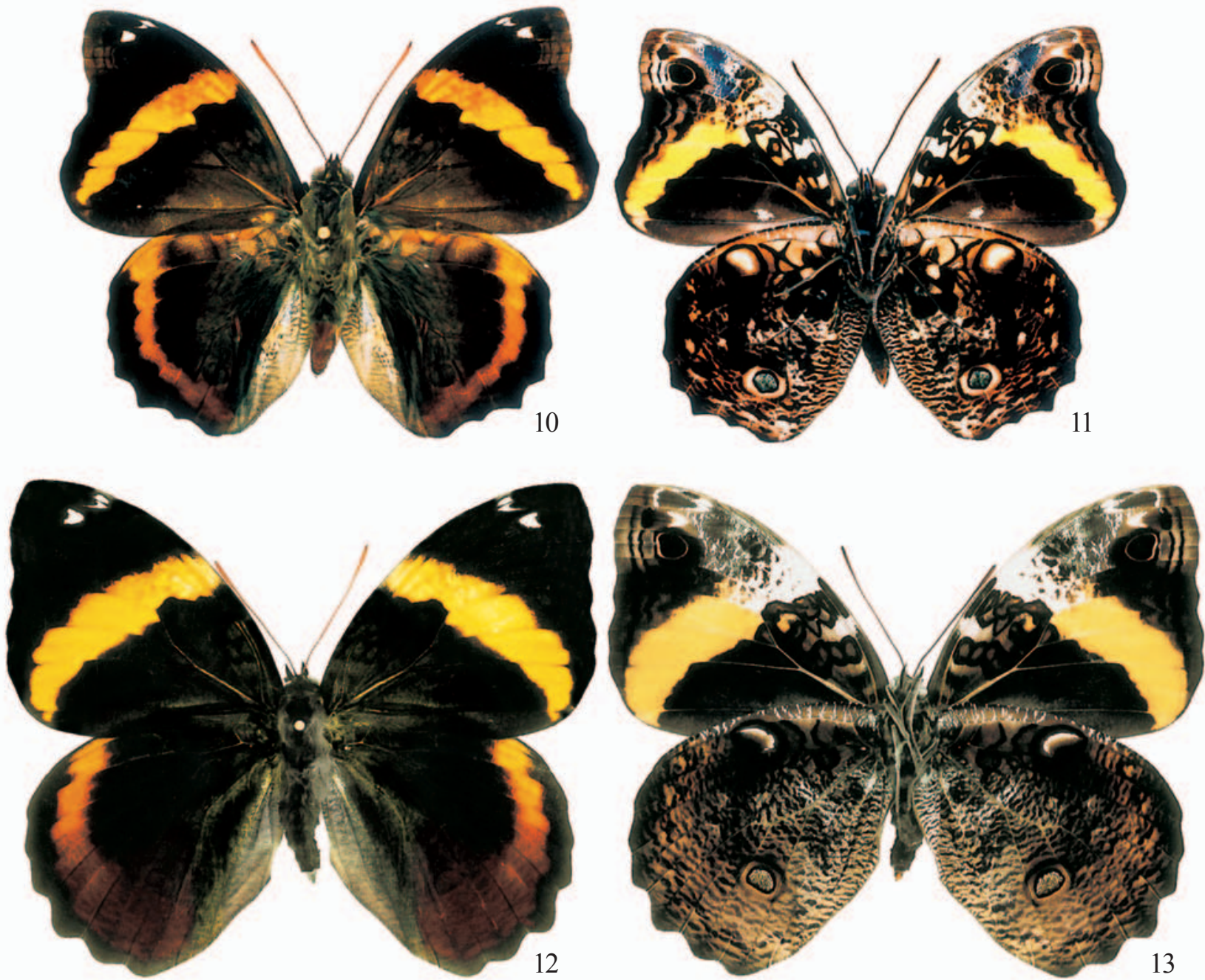

Figs. 10-13. Opsiphanes quiteria meridionalis Staudinger. Adulto. 10 , Macho vista dorsal; 11, Macho vista ventral; 12, Fêmea vista dorsal; 13, Fêmea vista ventral.

Pupa

(Figs. 7-9)

Coloração geral verde com linhas em castanho. Antenas, pernas e asas contornadas por tênues linhas castanhas. Três linhas castanhas margeadas de creme, formando uma espécie de carena estão assim distribuídas: a primeira, dorsal, desde o vértice, entre as antenas até o ápice do cremaster; tanto na região cefálica como no cremaster, apenas como uma linha de coloração distinta e entre estes pontos com limite acentuado. A segunda, também acentuada, forma o limite anterior da região cefálica e desce lateralmente até o segundo segmento abdominal, terminando em mancha arredondada de aspecto espelhado como citado para Caligo beltrao (Illiger, 1801) (Casagrande 1979); Caligo martia (Godart, [1824]); Dasyophthalma rusina rusina (Godart, [1824]); Dasyophthalma creusa creusa (Hübner, [1821]) (Casagrande \& Mielke 2000a,b, 2003). A terceira, mais ventral, com início próximo à mancha espelhada, acompanha a asa e continua apenas como linha de coloração mais escura sobre os espiráculos do quarto segmento abdomial até o cremaster. Duas manchas arredondadas castanhas, em posição ventral sobre as asas.

Abdome com linha mediana ventral desde o quarto segmento até o cremaster. Dorsalmente, linhas obliquoas percorrem os segmentos desde a linha que forma a carena medianamente até próximo aos espiráculos. Estas mesmas linhas foram descritas para Caligo telamonius memnon (C.Felder \& C.Felder, 1867) e Caligo atreus (Kollar, 1850) por Young \& Muyshondt 1985 (o primeiro como Caligo memnon) e para Caligo martia (Godart, [1824]) por Casagrande \& Mielke (2000a).

Atingem o comprimento máximo de $4 \mathrm{~cm}$ e largura máxima de $1,7 \mathrm{~cm}$.

Os adultos (Figs. 10-13) emergiram em novembro e dezembro, com um período na fase de pupa que variou entre 15 e 30 dias. 
Agradecimentos. Ao Sr. Ivo Rank por fornecer os exemplares estudados e por permitir o uso de seu borboletário em São Bento do Sul, Santa Catarina, para o desenvolvimento dos imaturos.

\section{REFERÊNCIAS}

Ackery, P. R. 1988. Hostplants and classification: a review of nymphalid butterflies. Biological Journal of the Linnean Society 33: 95203.

Ackery, P. R.; R. De Jong \& R. I. Vane-Wright. 1999. The butterflies: Hedyloidea, Hesperioidea and Papilionoidea, p. 263-300. In: N. P. Kristensen (ed.). 1999. Lepidoptera, Moths and Butterflies. Vol. 1: Evolution, Systematics, and Biogeography. In: M. Fischer (ed.). Handbook Zoology, Vol. 4. Berlin, New York. Walter de Gruyter, $491 \mathrm{p}$.

Casagrande, M. M. 1979. Sobre Caligo beltrao (Illiger). I: Taxonomia, Biologia, Morfologia das Fases Imaturas e Distribuições Espacial e Temporal (Lepidoptera, Satyridae, Brassolinae). Revista Brasileira Biologia 39: 173-193.

Casagrande, M. M. 2002. Naropini Stichel, taxonomia e imaturos (Lepidoptera, Nymphalidae, Brassolinae). Revista Brasileira de Zoologia 19: 467-569.

Casagrande, M. M. 2004. Tribe Brassolini, p. 201-204. In: G. Lamas (ed). Checklist: Part 4A, Hesperioidea-Papilionoidea, 439 p. In: J. Heppner. Atlas of Neotropical Lepidoptera. Association for Tropical Lepidoptera, Scientific Publishers. XXXVI + 439 p.

Casagrande, M. M. \& O. H. H. Mielke. 2000a. Larva de quinto estádio e pupa de Caligo martia (Godart) (Lepidoptera, Nymphalidae, Brassolinae). Revista Brasileira de Zoologia 17: 75-79.

Casagrande, M. M. \& O. H. H. Mielke. 2000b. Larva de quinto estádio e pupa de Dasyophthalma rusina rusina (Godart) (Lepidoptera, Nymphalidae, Brassolinae). Revista Brasileira de Zoologia 17: 401-404.

Casagrande, M. M. \& O. H. H. Mielke. 2003. Larvas de quarto e quinto estádios e pupa de Dasyophthalma creusa creusa (Hübner) (Lepidoptera, Nymphalidae, Brassolinae). Revista Brasileira de Zoologia 20: $157-160$.

De Vries, P. J. 1987. The Butterflies of Costa Rica and their Natural History. Papilionidae, Pieridae, Nymphalidae. 327 p. Princeton University Press.

Freitas, A. V. L. \& K. S. Brown Jr. 2004. Phylogeny of the Nymphalidae (Lepidoptera). Systematic Biology 53: 363-383.

Lamas, G. 2004. Checklist: Part 4A, Hesperioidea-Papilionoidea, 439 p. In: J. Heppner. Atlas of Neotropical Lepidoptera, Association for Tropical Lepidoptera, Scientific Publishers.

Yong, A. M. \& A. Muyshondt, 1985. Notes on Caligo memnon Felder and Caligo atreus Kollar. (Lepidoptera: Nymphalidae: Brassolinae) in Costa Rica and El Salvador. The Journal of Research on the Lepidoptera 24: 154-175. 